\section{Fatores associados ao acesso geográfico aos serviços de saúde por pessoas com tuberculose em três capitais do Nordeste brasileiro}

\author{
Factors associated with geographic access to \\ health services by TB patients in three State \\ capitals in Northeast Brazil
}

\section{Los factores asociados al acceso geográfico a los servicios de salud para personas con tuberculosis en tres capitales del nordeste brasileño}

\author{
1 Instituto de Saúde Coletiva, \\ Universidade Federal da \\ Bahia, Salvador, Brasil. \\ 2 Centro de Ciências da \\ Saúde, Universidade Federal \\ de Pernambuco, Recife, \\ Brasil. \\ 3 Centro de Pesquisas Aggeu \\ Magalhães, Fundação \\ Oswaldo Cruz, Recife, Brasil. \\ 4 Centro Biomédico, \\ Universidade do Estado do \\ Rio de Janeiro, Rio de Janeiro, \\ Brasil. \\ Correspondência \\ M. S. P. L. Souza \\ Rua Basílio da Gama s/n, \\ Campus Universitário \\ Canela, Salvador, $B A$ \\ 40110-040, Brasil. \\ marciasaopedrosouza@ \\ gmail.com
}

\begin{abstract}
The aim of this study was to identify factors associated with geographic access to health services by tuberculosis patients in three State capitals in Northeast Brazil. The sample consisted of new tuberculosis cases reported in 2007. The study used data from the Information System on Notifiable Diseases and the National Registry of Healthcare Organizations. Addresses of households and health services were geocoded, and difficult access was defined as a distance greater than 800 meters from the household to the health service. Crude prevalence ratios were estimated, as well as adjusted prevalence ratios using Poisson regression. After adjusting the study variables, the only variable that remained associated with difficult access was primary healthcare units in Salvador, Bahia State $(P R=0.75$; 95\%CI: 0.720-0.794) and in Recife, Pernambuco State (PR =0.402; 95\%CI: 0.318-0.508). The study concluded that decentralization of primary care can help improve access to health services.
\end{abstract}

Tuberculosis; Health Services Accessibility; Health Centers

\author{
Márcia São Pedro Leal Souza 1 \\ Rosana Aquino 1 \\ Susan M. Pereira 1 \\ Maria da Conceição Nascimento Costa ${ }^{1}$ \\ Mauricio Lima Barreto 1 \\ Marcio Natividade 1 \\ Ricardo Ximenes 2 \\ Wayner Souza ${ }^{3}$ \\ Odimariles Maria Dantas 2 \\ José Ueleres Braga ${ }^{4}$
}

\section{Resumo}

O objetivo do presente estudo foi identificar fatores associados ao acesso geográfico aos serviços de saúde por portadores de tuberculose em três capitais do Nordeste do Brasil. A amostra foi composta por casos novos de tuberculose, notificados em 2007. Foram utilizados dados provenientes do Sistema de Informação sobre Agravos de Notificação, e do Cadastro Nacional de Estabelecimento de Saúde. Os endereços dos domicílios e das unidades de saúde foram georreferenciados $e$, utilizando a distância entre o domicílio e a unidade de atendimento de cada caso, foi considerado acesso dificultado quando esta distância foi maior do que 800 metros. Foram estimadas as razões de prevalência bruta e ajustada por meio de regressão de Poisson. Verificou-se que após ajuste com as variáveis estudadas, apenas a variável unidade básica, em Salvador, Bahia ( $R P=0,75$; IC95\%: 0,720-0,794) $e$ em Recife, Pernambuco ( $R P=0,402$; IC95\%: 0,3180,508), manteve associação com o acesso dificultado. O estudo concluiu que a descentralização do atendimento em unidade básica pode contribuir com a melhoria do acesso aos serviços de saúde.

Tuberculose; Acesso aos Serviços de Saúde; Centros de Saúde 


\section{Introdução}

A ampliação do acesso à atenção à saúde, especialmente aos cuidados primários, para grande parte da população brasileira é um dos importantes avanços da implantação do Sistema Único de Saúde (SUS), que tem como princípios fundamentais a universalidade, a equidade e a participação social. Entretanto, ainda persistem grandes desafios para garantir o acesso universal e equitativo dos serviços de saúde, em um cenário nacional, marcado por profundas transformações demográficas, epidemiológicas e por desigualdades sociais 1

Os fatores socioeconômicos, como renda, escolaridade, local e condições de moradia, interferem no acesso aos serviços de saúde, favorecendo ou não a procura e utilização dos mesmos 2,3,4. Dentre as características dos sistemas e serviços de saúde que interferem diretamente nesse processo destacam-se: aquelas relativas à oferta, como disponibilidade de serviços e sua distribuição geográfica; disponibilidade e qualidade dos recursos humanos e tecnológicos; mecanismos de financiamento e modelo assistencial 3,5 .

Acentuadas desigualdades sociais entre regiões, assim como entre grupos populacionais, acarretam dificuldade no acesso aos serviços de saúde 6 e retardo no diagnóstico de diversas doenças, em especial, as doenças crônicas de início insidioso e de longa duração 7,8 . No caso da tuberculose, doença infecciosa cuja ocorrência está relacionada às condições de vida desfavoráveis, apesar da existência de tratamento conhecido e eficaz, a magnitude de sua morbidade e mortalidade ainda é elevada, particularmente nos países em desenvolvimento 9 . Assim, dificuldades no acesso aos serviços de saúde contribuem para o retardo do diagnóstico e tratamento da tuberculose o que, por sua vez, aumenta as possibilidades de abandono do tratamento, um dos principais obstáculos para o controle desta doença 10.

No Brasil, em 2012, a incidência de tuberculose foi de 35,8 casos por 100 mil habitantes 11 . As metas recomendadas pela Organização Mundial da Saúde de 70\% de detecção dos casos, $85 \%$ de cura utilizando a estratégia Tratamento Diretamente Observado (TDO) e 5\% de abandono, ainda não foram alcançadas pelo Brasil que apresentou no ano de 2010 porcentuais de cura de $70,3 \%$ e abandono de $10 \% 12$.

Embora o Programa Nacional de Controle da Tuberculose estabeleça em suas diretrizes a oferta do diagnóstico e tratamento de forma gratuita, desde 1971, estas ações indispensáveis para o seu controle ocorrem de forma desigual no território nacional, havendo entraves no acesso dos pacientes à atenção à saúde, devidos especialmente a aspectos organizacionais dos serviços de saúde 13,14.

Como há dificuldades no controle da tuberculose, preconiza-se que seu tratamento seja feito o mais próximo do local de moradia dos portadores, sempre que possível sob supervisão, visando a alcançar uma melhor adesão ao tratamento 9,15. Entretanto, no Brasil, o acesso aos serviços de saúde está relacionado à condição social dos indivíduos e do local onde residem, melhorando com o grau de desenvolvimento socioeconômico da região ${ }^{3}$. Assim sendo, é possível que pacientes com tuberculose residentes em regiões menos desenvolvidas deste país enfrentem dificuldades no acesso aos serviços de saúde.

$\mathrm{O}$ acesso é um conceito complexo que tem várias dimensões: organizacionais, sociais e geográficas, que se inter-relacionam. Neste trabalho, é abordada apenas a dimensão geográfica, que corresponde à localização dos serviços de saúde e pode ser medida pela distância linear entre o serviço de saúde e os domicílios dos pacientes 5,16.

O objetivo deste estudo é identificar os fatores associados ao acesso aos serviços de saúde por portadores de tuberculose em três capitais da Região Nordeste, Salvador (Bahia), Recife (Pernambuco) e João Pessoa (Paraíba), no ano de 2007.

\section{Material e método}

Realizou-se um estudo transversal envolvendo todos os casos novos de tuberculose, relativos a residentes em Salvador, Recife e João Pessoa, notificados no ano de 2007. Os dados sobre a doença foram obtidos no Sistema de Informação sobre Agravos de Notificação (SINAN) do Ministério da Saúde. Informações sobre as características das unidades de saúde notificantes foram provenientes do banco de dados do Cadastro Nacional de Estabelecimentos de Saúde (CNES).

As três capitais apresentavam características territoriais e populacionais distintas. A área territorial, população estimada e densidade populacional foram, respectivamente, $693,292 \mathrm{~km}^{2}$, 2.676.606 habitantes e 3.859,44 habitantes $/ \mathrm{km}^{2}$ em Salvador, $218,498 \mathrm{~km}^{2}, 1.536 .934$ habitantes e $7.039,64$ habitantes $/ \mathrm{km}^{2}$ em Recife e $211,474 \mathrm{~km}^{2}$, 723.514 habitantes e $3.421,28$ habitantes $/ \mathrm{km}^{2} \mathrm{em}$ João Pessoa (Instituto Brasileiro de Geografia e Estatística. Estados@. http://www.ibge.gov.br/ estadosat, acessado em 08/Mai/20114).

As variáveis de estudo relativas aos casos de tuberculose foram: características sociodemográficas (sexo, faixa etária, escolaridade, raça/ cor e endereço) dos indivíduos, forma clínica da 
doença, presença de comorbidade (alcoolismo, AIDS, diabetes e doença mental), indicação de TDO e ocorrência de abandono do tratamento. Dentre as variáveis relativas às unidades de saúde de atendimento foram consideradas: o endereço; natureza do serviço (público ou privado); tipo de estabelecimento (unidade básica, policlínica, hospitais); e esfera administrativa (federal, estadual, municipal e privada).

Os endereços das residências dos casos e das unidades de saúde de atendimento foram georreferenciados utilizando-se o Google Earth (https: //www.google.com/earth/), ferramenta de localização geográfica apenas para definir as coordenadas geográficas latitude e longitude. Após a exclusão de possíveis inconsistências, como pontos localizados fora dos limites dos municípios e coordenadas não localizadas em decorrência de endereços incompletos, o banco de dados espaciais foi visualizado pelo Sistema de Informação Geográfica ArcView 3.3 (Environmental Systems Research Institute Inc., http://www.esri. com/software/arcview/). Com bases nessas coordenadas, foram construídos buffers com raios definidos segundo a média das distâncias entre as residências e as unidades de saúde de atendimento, o que permitiu a construção do indicador de acesso dificultado, para classificar a distância entre a unidade de saúde notificante e o endereço do caso baseando-se na identificação das coordenadas geográficas e, a seguir, georreferenciados na malha do município. Os padrões de deslocamento dos casos foram analisados com base no cálculo de distância euclidiana representativa do deslocamento dos indivíduos, considerando-se quilômetro como unidade de distância nas três capitais. Esse cálculo corresponde a uma medida objetiva da distância entre o local de residência e o local de atendimento dos casos de tuberculose.

Para a definição do indicador acesso dificultado, foram realizadas análises da variável distância entre os serviços e os domicílios, e revisão da literatura de investigações que definissem padrões de distâncias para o deslocamento a pé de usuários de serviços públicos. Optou-se por criar a variável acesso dificultado como dicotômica, utilizando-se como ponto de corte da distância entre domicílio do caso de tuberculose e a unidade de saúde onde o caso foi atendido o valor de 800 metros, considerando que valores superiores a este limiar definiam o acesso dificultado. Essa definição foi baseada em estudos na área de urbanismo que apontam a distância de 800 metros como a percorrida pelo pedestre sem necessidade de acesso a transporte público, considerando ônibus e outros transportes urbanos 5,17, argumento de maior relevância do que a definição baseada na distribuição amostral da variável.
$\mathrm{Na}$ área da saúde, um tempo de deslocamento entre 5-35 minutos é considerado aceitável para um indivíduo se deslocar da sua residência até o serviço de saúde 18 , admitindo-se que em uma caminhada rápida de $5 \mathrm{~km} / \mathrm{hora}$, em 15 minutos seriam percorridos 1.250 metros. E considerando que o paciente em busca de atendimento não faz uma caminhada rápida, julgou-se que, em 15 minutos, seria razoável percorrer 800 metros.

Foram calculadas frequências simples, absolutas e relativas, das características do indivíduo e das unidades de saúde. Estimaram-se as proporções de casos de tuberculose de acordo com as diversas características selecionadas, considerando-se as distâncias entre a residência dos casos e as unidades de atendimento. As diferenças tiveram suas significâncias estatísticas apreciadas pelo teste qui-quadrado de Pearson, aceitando-se $\mathrm{p}<0,05$ como limite crítico.

Também foram calculadas médias, medianas e variação das distâncias percorridas (em km) pelos pacientes nos três municípios, e suas distribuições foram comparadas tendo como base a representação gráfica (Boxplot).

Para a análise multivariada foi utilizada a Regressão de Poisson, visando a obter a estimativa da razão de prevalência (RP) utilizando a estimativa robusta da variância, uma vez que nos casos em que a variável desfecho apresenta prevalência maior do que $10 \%$ a odds ratio (OR) obtida no modelo de regressão logística é uma estimativa válida da RP 19. Após a realização da análise univariada, as variáveis estatisticamente significantes $(\mathrm{p}<0,20)$, sexo, grupo etário, raça, escolaridade, agravos associados, serviço e unidade básica, foram selecionadas para a análise multivariada, em ordem da maior para a menor significância. A interpretação do modelo foi realizada em termos de RP, considerando um nível de 5\% de significância, utilizando o software Stata 10.0 (Stata Corp., College Station, Estados Unidos). Este projeto foi aprovado pelo Comitê de Ética do Instituto de Saúde Coletiva sob no 027-09.

\section{Resultados}

Em 2007, foram notificados 3.021 casos de tuberculose em Salvador, 1.106 em Recife e 399 em João Pessoa. Os valores da mediana da distância percorrida em quilômetros pelos casos de tuberculose até a unidade de saúde de atendimento foram similares em Salvador (mediana $=3,3$; $1,4 \mathrm{~km}-6,3 \mathrm{~km}$ ) e em João Pessoa (mediana $=3,7$; $2,1 \mathrm{~km}-6,0 \mathrm{~km}$ ) e menor em Recife (mediana $=1,3$; 0,5km-3,5km) (Figura 1). Os percentuais de acesso dificultado para Salvador, Recife e João Pessoa 
Descrição dos valores medianos da distância percorrida pelos casos de tuberculose até a unidade de saúde de atendimento em três capitais do Nordeste do Brasil: Salvador (Bahia); Recife (Pernambuco); João Pessoa (Paraíba), notificados em 2007

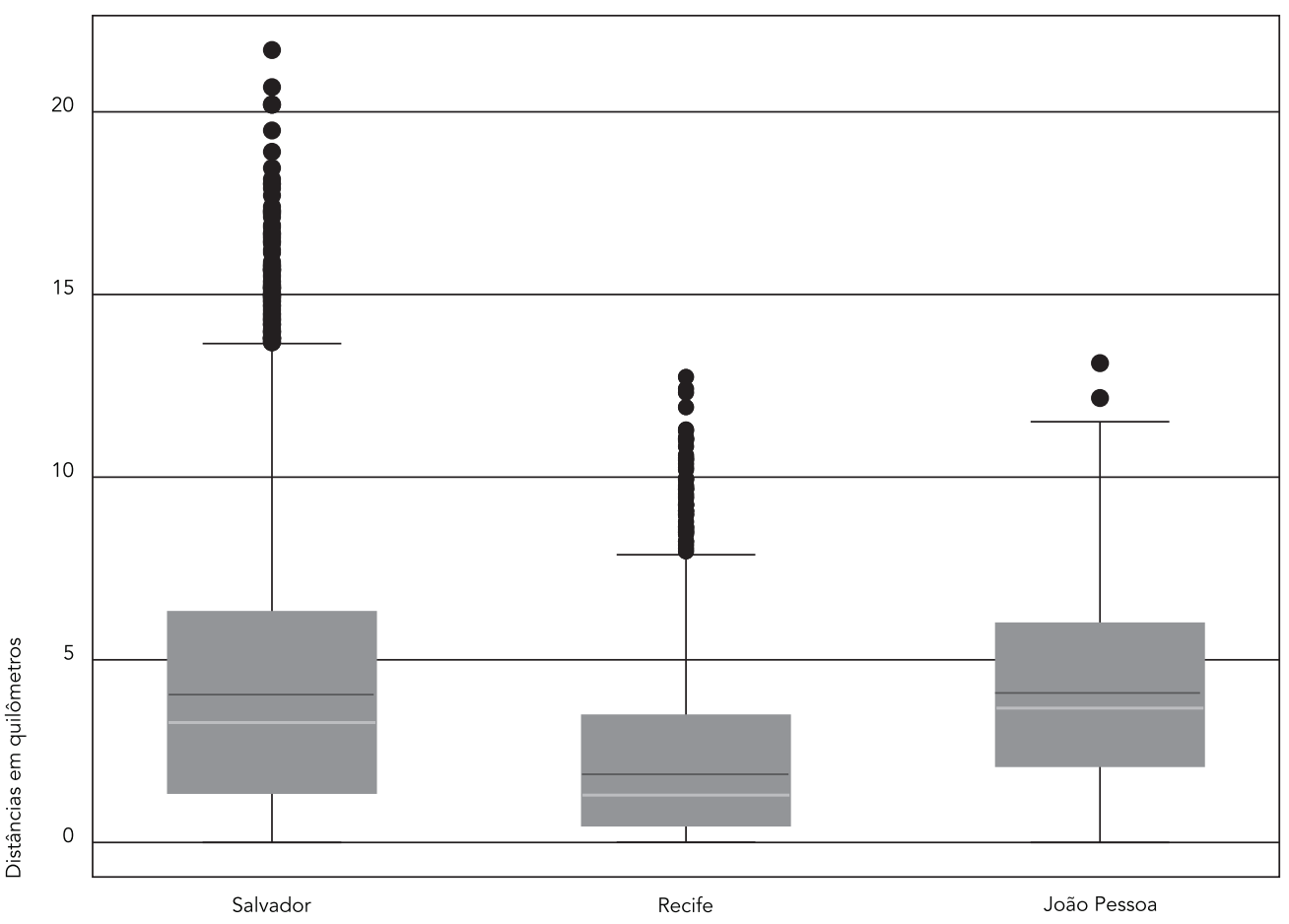

foram $84,6 \%, 62,9 \%$ e $90,9 \%$, respectivamente.

Em Salvador, mais de 50\% dos casos foram atendidos em três unidades de saúde: Hospital Especializado Octavio Mangabeira (37,7\% dos casos), Instituto Brasileiro para Investigação da Tuberculose - IBIT $(8,4 \%)$ e Unidade Básica de Saúde Ramiro de Azevedo (7,5\%). Em João Pessoa, $83,9 \%$ dos casos foram atendidos no Complexo Hospitalar Clementino Fraga. Em Recife, nenhuma das unidades atendeu mais de $8 \%$ dos casos, e as unidades que atenderam ao maior número de casos foram: Policlínica Lessa de Andrade (7,8\%), Policlínica Agamenon Magalhães $(7,4 \%)$, Policlínica Clementino Fraga $(6,1 \%)$ e Policlínica Albert Sabin (6,1\%).

Nas três capitais predominou o atendimento em unidades públicas de saúde (86\% em Salvador, $98,8 \%$ em Recife e $100 \%$ em João Pessoa). Em Salvador, 50\% dos atendimentos foram mais frequentes em hospitais e 41,4\% em unidades básicas; em João Pessoa os atendimentos em hospitais foram de $84 \%$; e em Recife, $45 \%$ dos atendimentos ocorreram em unidades básicas de saúde e apenas $17 \%$ em hospitais. A maioria das unidades de saúde em Salvador pertencia às esferas municipal $(41,4 \%)$ e estadual $(42,7 \%)$. Em Recife, $82,8 \%$ dos casos foram atendidos em unidades de saúde municipais e em João Pessoa, $84 \%$ na rede estadual. As diferenças foram estatisticamente significantes (Tabela 1).

Os percentuais de preenchimento das variáveis escolaridade e raça/cor foram $60,2 \%$ e $84,4 \%$, respectivamente. Não foram observadas diferenças estatisticamente significantes nos porcentuais de acesso dificultado segundo sexo idade, escolaridade e raça/cor nas três capitais. Em Recife, os percentuais de acesso dificultado foram maiores nos casos de tuberculose extrapulmonar, entre não alcoolistas, portadores de HIV/AIDS e aqueles que não tinham indicação de TDO. Em Salvador, apenas para a variável indicação de TDO as diferenças nos porcentuais de acesso dificultado foram estatisticamente significantes (Tabela 2).

Dentre as variáveis preditoras para o acesso dificultado, atendimento em unidade básica e 
Caracterização dos serviços de saúde segundo natureza do serviço, tipo de estabelecimento e esfera administrativa nos municípios de Salvador (Bahia), Recife (Pernambuco) e João Pessoa (Paraíba), Brasil, em 2007.

\begin{tabular}{|c|c|c|c|c|c|c|c|}
\hline \multirow[t]{2}{*}{ Variáveis } & \multicolumn{2}{|c|}{ Salvador $(n=3.021)$} & \multicolumn{2}{|c|}{ Recife $(n=1.106)$} & \multicolumn{2}{|c|}{ João Pessoa $(n=399)$} & \multirow[t]{2}{*}{ Valor de $p$ * } \\
\hline & $\mathbf{n}$ & $\%$ & $\mathbf{n}$ & $\%$ & $\mathbf{n}$ & $\%$ & \\
\hline \multicolumn{8}{|l|}{ Natureza do serviço } \\
\hline Público & 2.599 & 86,0 & 1.093 & 98,8 & 399 & 100,0 & 0,000 \\
\hline Privado & 422 & 14,0 & 13 & 1,2 & - & - & \\
\hline \multicolumn{8}{|c|}{ Tipo de estabelecimento } \\
\hline Unidade básica & 1.250 & 41,4 & 498 & 45,0 & 60 & 15,0 & 0,000 \\
\hline Policlínicas & 268 & 8,9 & 418 & 37,8 & 339 & 85,0 & \\
\hline Hospitais & 1.503 & 49,8 & 190 & 17,2 & - & - & \\
\hline \multicolumn{8}{|l|}{ Esfera administrativa } \\
\hline Municipal & 1.252 & 41,4 & 916 & 82,8 & 60 & 15,0 & 0,000 \\
\hline Estadual & 1.291 & 42,7 & 144 & 13,0 & 335 & 84,0 & \\
\hline Federal & 56 & 1,9 & 32 & 2,9 & 4 & 1,0 & \\
\hline
\end{tabular}

* Utilizado o teste estatístico do qui-quadrado de Pearson para o cálculo do valor de $p$, aceitando-se $p<0,05$ como limite crítico.

natureza do serviço público/privado, apresentaram RP brutas com efeito significante para Salvador; para Recife foram as comorbidades, público/privado e unidade básica; e em João Pessoa apenas a variável unidade básica. Entretanto, após ajuste com todas as outras variáveis analisadas, somente a variável unidade básica, em Salvador $(\mathrm{RP}=0,756$; intervalo de $95 \%$ de confiança, IC95\%: 0,720-0,794) e em Recife (RP = 0,402; IC95\%: 0,318-0,508), manteve a significância estatística, sugerindo que ser atendido em unidade básica é um fator que facilita o acesso dos pacientes à atenção (Tabela 3 ).

\section{Discussão}

No presente trabalho, a perda de endereços não mapeados foi menor do que $7 \%$, o que não deve ter influenciado nos cálculos das distâncias médias dos pacientes estudados. Entretanto, como é provável que endereços não georreferenciados sejam de mais difícil acesso, as médias podem estar subestimadas e as diferenças podem ser maiores do que as encontradas. Cabe ressaltar que a direção desse possível viés é no sentido de reafirmar nossos resultados.

$\mathrm{O}$ atendimento dos casos de tuberculose em Salvador e João Pessoa estava centralizado em poucas unidades de referência, e em Recife o atendimento na maioria dos casos estava descentralizado em unidades básicas. Não houve associação entre as variáveis sexo, idade, escola- ridade, raça/cor, agravos associados e acesso dificultado. $\mathrm{O}$ atendimento em unidade básica foi a única variável estatisticamente significante dos modelos. Em Salvador e Recife o acesso dificultado foi menor nesse grupo, já em João Pessoa houve um pequeno aumento no acesso dificultado.

O predomínio da assistência em unidades de referência em Salvador e João Pessoa pode ser explicado pela presença de hospitais com forte influência na atenção ao tratamento da tuberculose, o que evidencia a centralização das ações relacionadas à tuberculose, em contradição com o preconizado pelo Ministério da Saúde. Em João Pessoa, a magnitude do processo de centralização é maior do que nas demais capitais estudadas, dificultando o acesso ao serviço. A literatura afirma que a descentralização do tratamento dos casos de tuberculose favorece o acesso dos pacientes aos serviços de saúde, bem como facilita a busca ativa dos sintomáticos respiratórios, a coleta de escarro para a baciloscopia e a detecção dos casos pulmonares bacilíferos 20 .

Em Salvador, metade dos casos de tuberculose foi atendida em hospitais, apontando para a concentração da atenção neste nível do sistema. Uma explicação plausível para esse achado é a presença de hospitais de referência com forte influência na atenção ao tratamento da tuberculose. Desse modo, constata-se que nesse município não é atendida a orientação do Ministério da Saúde que preconiza que o tratamento com Esquema 1 (2RHZ/4RH), indicado para casos novos, deve ser realizado em nível ambulatorial 21 , 
Tabela 2

Percentual de casos de tuberculose nos municípios de Salvador (Bahia), Recife (Pernambuco) e João Pessoa (Paraíba) em relação à distância da residência até a unidade de atendimento segundo sexo, faixa etária, escolaridade, raça, forma, agravos associados e indicação de tratamento diretamente observado.

\begin{tabular}{|c|c|c|c|c|c|c|c|c|c|}
\hline \multirow[t]{2}{*}{ Variáveis } & \multicolumn{3}{|c|}{ Salvador (\%) } & \multicolumn{3}{|c|}{ Recife (\%) } & \multicolumn{3}{|c|}{ João Pessoa (\%) } \\
\hline & $\mathrm{n}$ & $\begin{array}{c}\text { Acesso } \\
\text { dificultado }\end{array}$ & Valor de p & $\mathbf{n}$ & $\begin{array}{c}\text { Acesso } \\
\text { dificultado }\end{array}$ & Valor de $p$ & $\mathrm{n}$ & $\begin{array}{c}\text { Acesso } \\
\text { dificultado }\end{array}$ & Valor de $p$ \\
\hline \multicolumn{10}{|l|}{ Sexo } \\
\hline Masculino & 1.876 & 85,5 & 0,069 & 480 & 62,9 & 0.212 & 224 & 92,0 & 0.338 \\
\hline Feminino & 1.150 & 83,0 & & 291 & 67,4 & & 126 & 88,9 & \\
\hline \multicolumn{10}{|l|}{ Faixa etária (anos) } \\
\hline $0<15$ & 133 & 82,7 & 0,657 & 46 & 82,6 & 0,078 & 20 & 95,0 & 0,885 \\
\hline $15<30$ & 1.052 & 83,9 & & 208 & 62,0 & & 114 & 91,2 & \\
\hline $30<40$ & 630 & 86,5 & & 185 & 64,9 & & 81 & 91,4 & \\
\hline $40<50$ & 578 & 84,3 & & 156 & 59,6 & & 72 & 91,7 & \\
\hline $50-60$ & 357 & 85,4 & & 107 & 65,4 & & 36 & 88,9 & \\
\hline$>60$ & 276 & 83,0 & & 404 & 60,6 & & 27 & 85,2 & \\
\hline \multicolumn{10}{|c|}{ Escolaridade (anos de estudo) } \\
\hline$<4$ & 681 & 84,6 & 0.797 & 132 & 54,5 & 0.400 & 108 & 91,7 & 0,756 \\
\hline $4-7$ & 671 & 83,8 & & 121 & 55,4 & & 75 & 92,0 & \\
\hline $8-11$ & 402 & 82,8 & & 51 & 64,7 & & 103 & 88,4 & \\
\hline 12 ou mais & 281 & 85,4 & & 41 & 65,9 & & 17 & 94,1 & \\
\hline \multicolumn{10}{|l|}{ Raça } \\
\hline Branca & 366 & 85,0 & 0,198 & 160 & 68,7 & 0,344 & 95 & 85,3 & 0,073 \\
\hline Preta & 859 & 85,5 & & 103 & 58,3 & & 29 & 93,1 & \\
\hline Parda & 1.503 & 84,1 & & 340 & 64,7 & & 217 & 93,5 & \\
\hline Outras & 66 & 75,8 & & 35 & 60,0 & & 4 & 75,0 & \\
\hline \multicolumn{10}{|l|}{ Forma } \\
\hline Pulmonar & 2.609 & 84,4 & 0,332 & 632 & 61,5 & 0,000 & 302 & 90,7 & 0,834 \\
\hline Extrapulmonar & 387 & 86,3 & & 138 & 78,3 & & 48 & 91,7 & \\
\hline \multicolumn{10}{|l|}{ Alcoolismo } \\
\hline Sim & 437 & 87,9 & 0.195 & 129 & 58,1 & 0,001 & 44 & 90,9 & 0,898 \\
\hline Não & 1.475 & 85,4 & & 405 & 74,1 & & 247 & 91,5 & \\
\hline \multicolumn{10}{|l|}{ AIDS } \\
\hline $\operatorname{Sim}$ & 129 & 89,2 & 0,112 & 62 & 87,1 & 0,002 & 27 & 96,3 & 0,312 \\
\hline Não & 1.190 & 83,8 & & 418 & 68,2 & & 219 & 90,4 & \\
\hline \multicolumn{10}{|l|}{ Diabetes } \\
\hline Sim & 190 & 84,2 & 0,479 & 48 & 68,8 & 0,790 & 24 & 100,0 & 0,127 \\
\hline Não & 1.697 & 86,1 & & 476 & 70,6 & & 269 & 91,1 & \\
\hline \multicolumn{10}{|l|}{ Doença mental } \\
\hline $\operatorname{Sim}$ & 43 & 81,4 & 0,368 & 24 & 58,3 & 0,186 & 8 & 100,0 & 0,388 \\
\hline Não & 1.805 & 86,2 & & 499 & 70,9 & & 281 & 91,5 & \\
\hline \multicolumn{10}{|c|}{ Indicação tratamento } \\
\hline \multicolumn{10}{|c|}{ diretamente observado } \\
\hline Sim & 390 & 72,8 & 0,000 & 357 & 54,6 & 0,000 & 135 & 88,9 & 0,220 \\
\hline Não & 2.417 & 86,9 & & 288 & 78,5 & & 194 & 92,8 & \\
\hline
\end{tabular}


Resultados da análise multivariada por regressão de Poisson para a razão de prevalência de acesso dificultado, segundo sexo, grupo etário, raça, escolaridade, agravos associados, tipo de serviço e tipo de unidade.

\begin{tabular}{|c|c|c|c|c|c|c|}
\hline \multirow[t]{3}{*}{ Variáveis } & \multirow{2}{*}{\multicolumn{2}{|c|}{$\begin{array}{l}\text { Salvador } \\
\text { Modelo } 1\end{array}$}} & \multirow{2}{*}{\multicolumn{2}{|c|}{$\begin{array}{c}\text { Recife } \\
\text { Modelo } 2\end{array}$}} & \multirow{2}{*}{\multicolumn{2}{|c|}{$\begin{array}{c}\text { João Pessoa } \\
\text { Modelo } 3\end{array}$}} \\
\hline & & & & & & \\
\hline & RP & IC95\% & $\mathrm{RP}$ & IC95\% & RP & IC95\% \\
\hline \multicolumn{7}{|l|}{ Sexo } \\
\hline masculino & 1,017 & $0,976-1,060$ & 0,893 & $0,763-1,044$ & 1,011 & $0,934-1,095$ \\
\hline \multicolumn{7}{|l|}{ Grupo etário } \\
\hline$\geq 30$ anos & 0,992 & $0,955-1,030$ & 0,960 & $0,814-1,132$ & 0,986 & $0,911-1,066$ \\
\hline \multicolumn{7}{|l|}{ Raça } \\
\hline Não branca & 1,000 & $0,963-1,040$ & 0,903 & $0,763-1,068$ & 0,966 & $0,872-1,069$ \\
\hline \multicolumn{7}{|l|}{ Escolaridade * } \\
\hline Baixa escolaridade & 1,011 & $0,992-1.029$ & 0,915 & $0,775-1,082$ & 1,022 & $0,951-1,098$ \\
\hline \multicolumn{7}{|l|}{ Agravos associados ** } \\
\hline Sim & 0,999 & $0,956-1,045$ & 0,882 & $0,750-1,039$ & 1,032 & $0,953-1,117$ \\
\hline \multicolumn{7}{|l|}{ Serviço } \\
\hline Público & 1,038 & $0,992-1,086$ & - & - & - & - \\
\hline \multicolumn{7}{|l|}{ Unidade Básica } \\
\hline Sim & 0,756 & $0,720-0,794$ & 0,402 & $0,318-0,508$ & 1,084 & $1,013-1,160$ \\
\hline
\end{tabular}

IC95\%: intervalo de 95\% de confiança.

* Escolaridade: baixa escolaridade - menor que 4 anos de estudos;

** Agravos associados (alcoolismo, HIV/AIDS, diabetes, doença mental).

Nota: $p<0,005$ para a variável unidade básica em Salvador (Bahia) e Recife (Pernambuco).

no local mais próximo à residência do paciente, ficando a indicação de internação reservada para os casos específicos: meningite tuberculosa, indicações cirúrgicas em decorrência da tuberculose, complicações graves, intolerância medicamentosa incontrolável em ambulatório, intercorrências clínicas ou cirúrgicas graves ${ }^{9}$.

Em Recife, o percentual dos atendimentos em unidades básicas foi superior quando comparado com as demais capitais, o que indica uma atenção à saúde descentralizada. A literatura afirma que a atenção básica é responsável pela distribuição mais equânime de saúde entre populações 22 . O predomínio do atendimento dos casos de tuberculose em unidades públicas é esperado, na medida em que esta é uma patologia crônica, cujo tratamento é totalmente financiado pelo estado 22,23 .

A literatura aponta nível socioeconômico, sexo, idade, cor da pele, presença de companheiro, condições de saúde, existência de procura regular por serviços de saúde como fatores associados à utilização destes serviços 4,24. Pesquisas relatam que mulheres utilizam mais os serviços de saúde do que os homens e que a procura pelo serviço depende da renda familiar e do status social individual 25. Entretanto, no presente trabalho, as va- riavéis sexo e raça não apresentam significância estatística em relação ao acesso dificultado aos serviços de saúde. Diferenças estatisticamente não significantes nos percentuais de acesso dificultado no que se refere às variáveis sexo, idade e escolaridade dos casos de tuberculose nas três capitais, sugerem que estas variáveis não foram relevantes para a explicação do problema na população estudada e que outros fatores não observados no trabalho podem influenciar.

Sabe-se que a condição socioeconômica interfere na procura por serviços de saúde especializados; indivíduos de baixa renda terão dificuldade em se deslocar para os serviços de saúde mais distantes. Nas três capitais estudadas, uma hipótese para a não relação entre os fatores socioeconômicos analisados e o acesso dificultado ao serviço de saúde encontra-se na centralização do Programa de Controle da Tuberculose em unidades e hospitais especializados de referência para o tratamento.

A predominância de menores de 15 anos , em Recife e João Pessoa, com atendimentos em unidades de saúde situadas a uma distância superior a $800 \mathrm{~m}$, evidencia um padrão de acesso que prioriza unidades de referência no tratamento da tuberculose, como ocorre em João Pessoa com o 
atendimento no Complexo Hospitalar Clementino Fraga, o que favorece o processo de centralização no tratamento da tuberculose. Uma explicação para o acesso dificultado nessa faixa etária está na realização da busca ativa dos casos no ambulatório do Complexo Hospitalar Clementino Fraga, que tem o objetivo de resgatar, em suas próprias residências, os pacientes de tuberculose que abandonam o tratamento.

Uma provável explicação para uma elevada frequência de atendimentos em unidades mais distantes da residência, independentemente do nível de escolaridade em Salvador, Recife e João Pessoa, pode estar na compreensão do indivíduo em relação à doença, seu estado de saúde, estigma da doença e qualidade no serviço de saúde, levando-o a procurar por serviços de referência no tratamento da tuberculose 2 . Logo, variações geográficas, sociais, necessidades individuais, qualidade de vida, nível de conhecimento sobre saúde, associandos a fatores como sexo, idade, morbidade e condições econômicas, interferem no uso dos serviços de saúde 23 .

Na presença de agravo associado à tuberculose (alcoolismo, HIV, diabetes, doença mental), é importante o acompanhamento em um serviço de saúde especializado, pela demanda de exames específicos. Nas três capitais, os indivíduos com comorbidades associadas foram atendidos distantes da sua residência, o que sugere uma preocupação na procura de serviço de saúde especializado para um melhor diagnóstico, tratamento e acompanhamento. O uso excessivo do álcool e a presença do HIV apresentaram associação significante com acesso dificultado no Município de Recife, ratificando a necessidade de tratamento em serviços especializados.

Acesso dificultado mostrou-se estatisticamente significante para as variáveis indicação de TDO em Salvador, e para forma clínica, álcool, HIV/AIDS e TDO em Recife. Essa estratégia de tratamento (TDO) visa a garantir a conclusão do tratamento e evitar a multidrogaresistência, e consiste na administração direta do medicamento por um profissional de saúde ou uma segunda pessoa, devidamente treinada 26. Maiores porcentuais de acesso dificultado foram observados entre os pacientes com indicação de TDO nos municípios de Salvador e Recife, e podem ser explicados pela procura de serviços de referência para o tratamento da tuberculose por parte dos indivíduos, mesmo que para isto se- ja necessário percorrer maiores distâncias das suas residências.

Por ter sido realizado com base em dados secundários, uma das limitações do presente trabalho é a qualidade dos dados e a restrição do elenco das principais variáveis estudadas. Entretanto, as variáveis disponíveis no banco como sexo, faixa etária, escolaridade, raça, forma da doença, comorbidades, indicação de TDO, tipo de serviço de saúde e unidade básica eram relevantes para a análise do acesso geográfico aos serviços de saúde, considerando as características distintas das três capitais. Outro fator a ser destacado é a abordagem metodológica do acesso aos serviços de saúde, que neste trabalho foi considerado apenas o acesso geográfico, visto que outros fatores como acolhimento e organização dos serviços de saúde também contribuem para a determinação do acesso. Finalmente, a variável de acesso dificultado poderia ter sido analisada como variável contínua. Entretanto, a opção por um ponto de corte baseado na literatura disponível estabelece um padrão, o que pode contribuir para a comparação entre estudos que investiguem esta temática.

Salienta-se que na literatura não há um consenso quanto ao conceito de acesso dificultado, nem da distância geográfica aceitável entre a residência dos casos e a unidade de saúde de atendimento. A abordagem deste estudo pode ser considerada como inovadora porque permitiu, por meio de dados secundários, esclarecer alguns aspectos referentes ao acesso aos serviços de saúde em três capitais brasileiras. Ter sido atendido em unidade básica foi um fator de proteção para acesso dificultado nos municípios de Salvador e Recife. A relevância do presente estudo está na possibilidade de estabelecer "parâmetros espaciais” para caracterizar possíveis fatores associados à dificuldade de acesso a serviço de saúde para o tratamento da tuberculose. Entende-se que para se obter resposta efetiva no tratamento da tuberculose é necessário não só a transferência dos serviços de atenção à saúde para a atenção básica, mas também a estruturação das unidades de saúde para atender de forma integral os pacientes. Porém, no Brasil, o processo de descentralização para o controle da tuberculose tem sido extremamente lento e complexo, e a distância geográfica entre os serviços de saúde e a residência dos pacientes constitui um fator limitante. 


\section{Resumen}

El objetivo de este estudio fue identificar los factores asociados con el acceso geográfico a los servicios de salud para pacientes con tuberculosis en tres capitales del Nordeste. La muestra constó de nuevos casos de tuberculosis en 2007. El estudio usó datos del Sistema de Información de Enfermedades de Notificación Obligatoria y del Registro Nacional de Establecimientos de Salud. Las direcciones de los hogares y establecimientos de salud fueron geocodificados y, utilizando la distancia entre el hogar y la unidad de cuidados en cada caso, se consideró de difícil acceso cuando esta distancia era mayor de 800 metros. Se calcularon los cocientes de brutos y ajustados mediante la regresión de Poisson por razones de prevalencia. Se encontró que después de ajustar las variables estudiadas, sólo la unidad variable básica en Salvador ( $R P=0,75$; IC95\%: 0.720-0.794) $y$ Recife (OR = 0,402; IC95\%: 0,318-0,508) se mantuvieron asociadas con difícil acceso. La conclusión es que la descentralización de la atención en las unidades básicas de atención puede contribuir a la mejora del acceso a los servicios de salud.

Tuberculosis; Accesibilidad a los Servicios de Salud; Centros de Salud

\section{Colaboradores}

M. S. P. L. Souza participou da concepção e elaboração do projeto da obra, realizou a análise, interpretação dos resultados e a redação do artigo. R. Aquino participou da concepção e elaboração do projeto da obra, realizou a análise e interpretação dos resultados, a redação do artigo e foi responsável pela revisão crítica do conteúdo intelectual. S. M. Pereira participou em conjunto da concepção e elaboração do projeto da obra e realizou a análise e interpretação dos resultados, a redação do artigo e foi responsável pela revisão crítica do conteúdo intelectual. M. C. N. Costa participou em conjunto da concepção e elaboração do projeto da obra, realizou a análise e interpretação dos resultados e foi responsável pela revisão crítica do conteúdo intelectual. M. L. Barreto participou em conjunto da concepção e elaboração do projeto da obra e realizou a análise e interpretação dos resultados, a redação do artigo e foi responsável pela revisão crítica do conteúdo intelectual. M. Natividade participou em conjunto da concepção e elaboração do projeto da obra, realizou a análise, interpretação dos resultados e a análise espacial e redação do método. R. Ximenes participou em conjunto da concepção e elaboração do projeto da obra e realizou a análise e interpretação dos resultados, a redação do artigo e foi responsável pela revisão crítica do conteúdo intelectual. W. Souza participou em conjunto da concepção e elaboração do projeto da obra, realizou a análise e interpretação dos resultados, a redação do artigo e foi responsável pela revisão crítica do conteúdo intelectual. O. M. Dantas participou em conjunto da concepção e elaboração do projeto da obra e realizou a análise e interpretação dos resultados, bem como a redação do artigo e foi responsável pela revisão crítica do conteúdo intelectual. J. U. Braga participou em conjunto da concepção e elaboração do projeto da obra, realizou a análise, interpretação dos resultados, a análise espacial e a redação do método; foi responsável pela revisão crítica do conteúdo intelectual.

\section{Referências}

1. Paim J, Travassos C, Almeida C, Bahia L, Macinko J. O sistema de saúde brasileiro: história, avanços e desafios. The Lancet 2011; Series:11-31. http:// download.thelancet.com/flatcontentassets/pdfs/ brazil/brazilpor1.pdf (acessado em 19/Set/2012).

2. Motta CPG, Fahel MCX, Pimentel EC. Desigualdades no acesso aos serviços de saúde da população ocupada: uma análise a partir dos dados das PNADS 1998 e 2003. In: XVI Congresso da Associação Brasileira de Estudos Populacionais. Caxambu: Associação Brasileira de Estudos Populacionais; 2008. p. 1-21.
3. Travassos C, Oliveira EXG, Viacava F. Desigualdades geográficas e sociais no acesso aos serviços de saúde no Brasil: 1998 e 2003. Ciênc Saúde Coletiva 2006; 11:975-86.

4. Castro MSM, Travassos C, Carvalho MS. Efeito da oferta de serviços de saúde no uso de internações hospitalares no Brasil. Rev Saúde Pública 2005; 39:277-84.

5. Cunha A, Vieira-da-Silva LM. Acessibilidade aos serviços de saúde em um município do Estado da Bahia, Brasil, em gestão plena do sistema. Cad Saúde Pública 2010; 26:725-37. 
6. Klugman J. Relatório do Desenvolvimento Humano 2011. Sustentabilidade e equidade: um futuro melhor para todos. New York: Programa das Nações Unidas para o Desenvolvimento; 2011.

7. Chern JPS, Chen DR, Wen TH. Delayed treatment of diagnosed pulmonary tuberculosis in Taiwan. BMC Public Health 2008; 8:236.

8. Marmot M. Health in an unequal world. Lancet 2006; 368:2081-94

9. Perrechi MCT, Ribeiro SA. Tratamento de tuberculose: integração entre assistência hospitalar e rede básica na Cidade de São Paulo. J Bras Pneumol 2009; 35:1100-6.

10. Storla DG, Yimer S, Bjune GA. A systematic review of delay in the diagnosis and treatment of tuberculosis. BMC Public Health 2008; 8:15.

11. Ministério da Saúde. O controle da tuberculose no Brasil: avanços, inovações e desafios. http:// portalsaude.saude.gov.br/portalsaude/noticia/ 4567/162/tuberculose:-brasil-reduz-numero-decasos-em-354-no-ultimo-ano.html (acessado em 19/Set/2012).

12. Coordenação Geral de Doenças Endêmicas, Departamento de Vigilância Epidemiológica, Secretaria de Vigilância em Saúde, Ministério da Saúde. Programa Nacional de Controle da Tuberculose. Plano Estratégico para o Controle da Tuberculose, Brasil 2007-2015. http://www.paho.org/bra/in dex2.php?option=com_docman\&task=doc_view\&gi $\mathrm{d}=927 \&$ Itemid $=423$ (acessado em $22 / \mathrm{Mai} / 2014$ )

13. Scatena LM, Villa TCS, Ruffino Netto AR. Dificuldades de acesso a serviços de saúde para diagnóstico de tuberculose em municípios do Brasil. Rev Saúde Pública 2009; 43:389-97.

14. Figueiredo TMRM, Villa TCS, Scatena LM, Gonzales RIC, Ruffino-Netto A, Nogueira JA, et al. Desempenho da atenção básica no controle da tuberculose. Rev Saúde Pública 2009; 43:825-31.

15. Gonzales ICG, Monroe AA, Assis EG, Palha PF, Villas TCS, Ruffino Neto A. Desempenho de serviços de saúde no tratamento diretamente observado no domicílio para controle da tuberculose. Rev Esc Enferm USP 2008; 42:628-34.

16. Travassos C, Martins M. Uma revisão sobre os conceitos de acesso e utilização de serviços de saúde. Cad Saúde Pública 2004; 20 Suppl 2:S190-8.
17. Campos Filho CM. Reinvente o seu bairro. Caminhos para você participar do planejamento de sua cidade. São Paulo: Editora 34; 2003.

18. Ramos DD, Lima MADS. Acesso e acolhimento aos usuários em uma unidade de saúde de Porto Alegre, Rio Grande do Sul, Brasil. Cad Saúde Pública 2003; 19:27-34.

19. McCullagh P, Nelder JA. Generalized linear models. 2nd Ed. Boca Raton: Chapman and Hall; 1989.

20. Amaral AS, Tamaki EM, Sales CM, Renovato RD. Avaliação da descentralização do programa de controle da tuberculose do nível secundário para o nível primário do sistema de saúde de DouradosMS. Saúde Soc 2010; 19:794-802.

21. Departamento de Vigilância Epidemiológica, Secretaria de Vigilância em Saúde, Ministério da Saúde. Manual de recomendações para o controle da tuberculose no Brasil. Brasília: Ministério da Saúde; 2011.

22. Starfield B, Shi L, Macinko J. Contribution of primary care to health systems and health. Milbank Q 2005; 83:457-502.

23. Dias-da-Costa JS, Presser AD, Zanolla AF, Ferreira DG, Perozzo G, Freitas IBA, et al. Utilização dos serviços ambulatoriais de saúde por mulheres: estudo de base populacional no Sul do Brasil. Cad Saúde Pública 2008; 24:2843-51.

24. Sawyer DO, Leite IC, Alexandrino R. Perfis de utilização de serviços de saúde no Brasil. Ciên Saúde Coletiva 2002; 7:757-76.

25. Travassos C, Viacava F, Pinheiro R, Brito A. Utilização dos serviços de saúde no Brasil: gênero, características familiares e condição social. Rev Panam Salud Pública 2002; 11:365-73.

26. Departamento de Vigilância Epidemiologica, Secretaria de Vigilancia em Saúde, Ministério da Saúde. Tratamento diretamente observado (TDO) da tuberculose na atenção básica: protocolo de enfermagem Brasília: Ministério da Saúde; 2011. (Série F. Comunicação e Educação em Saúde).

Recebido em 21/Jan/2014

Versão final reapresentada em 18/Jul/2014

Aprovado em 29/Ago/2014 